\title{
Using Invariant Image Features for Synchronization in Spread Spectrum Image Watermarking
}

\author{
Ebroul Izquierdo \\ Multimedia and Vision Research Lab, Department of Electronic Engineering, Queen Mary, University of London, London, UK \\ Email: ebroul.izquierdo@elec.qmul.ac.uk
}

Received 10 August 2001 and in revised form 10 December 2001

\begin{abstract}
A watermarking scheme is presented in which the characteristics of both spatial and frequency techniques are combined to achieve robustness against image processing and geometric transformations. The proposed approach consists of three basic steps: estimation of the just noticeable image distortion, watermark embedding by adaptive spreading of the watermark signal in the frequency domain, and extraction of relevant information relating to the spatial distribution of pixels in the original image. The just noticeable image distortion is used to insert a pseudo-random signal such that its amplitude is maintained below the distortion sensitivity of the pixel into which it is embedded. Embedding the watermark in the frequency domain guarantees robustness against compression and other common image processing transformations. In the spatial domain most salient image points are characterized using the set of Hilbert first-order differential invariants. This information is used to detect geometrical attacks in a frequency-domain watermarked image and to resynchronize the attacked image. The presented schema has been evaluated experimentally. The obtained results show that the technique is resilient to most common attacks including rotation, translation, and scaling.
\end{abstract}

Keywords and phrases: watermarking, data hiding, image invariants.

\section{INTRODUCTION}

Conventional analog media distribution systems have an inherent built-in defense against copying, alteration, and fraud. Each time a new copy is issued the quality of the duplicated content is degraded accordingly. In contrast to that, digital multimedia documents are completely susceptible to exact replication and alteration. This, together with the rapid proliferation of digital documents, multimedia processing tools and the world-wide availability of internet access have created an ideal medium for piracy, copyright fraud, and uncontrollable distribution of high quality but unregistered multimedia content. Since digital watermarking can be seen as a solution to this problem, both the number of activities in this area and the recognition of the difficulties and challenges involved in this new technology have increased in the last few years $[1,2]$. Basically, the major challenge is to find a strategy that satisfies the conflicting objectives of performing image changes that are imperceptible for the human eye and being extremely robust against detection or removal either accidentally or intentionally. These two objectives are conflicting in nature because it is not possible to simultaneously maximize robustness and imperceptibility. Indeed, maximization of robustness leads to the introduction of large distortions in the image or video and consequently strong perceivable image changes. On the other hand, keeping imperceptibility means keeping the embed amount of information minimal and consequently very susceptible to removal or detection.

Usually digital watermarks are classified according to the embedding and retrieval domain, that is, the transform coefficient magnitude in the frequency domain and the luminance intensity in the spatial domain. Frequency-based techniques are very robust against certain kinds of signal processing, such as compression and filtering $[3,4]$. Since the watermark is spread throughout the image data, rather than targeting individual pixels, any attempts at attack means that the most fundamental structural components of the data must be targeted. This increases the chances of fidelity degradation, thereby rendering the attacked image useless. Using common image processing operations, if image integrity has not been compromised, the watermark can be reliably detected. In this context many watermarking algorithms relying on spread spectrum and different transform domains have been proposed in the literature: using the discrete wavelet transform (DWT) [5], the discrete Cosine transform (DCT) $[3,6]$, the discrete Fourier transform (DFT) [4], and so forth.

However, reliable detection of the frequency-based watermark is impeded when synchronization is lost as a result of geometric transformations. Although watermarking using spread spectrum in a transformed domain as described before is very resistant to amplitude distortions and additive noise, it becomes fragile if the starting point 


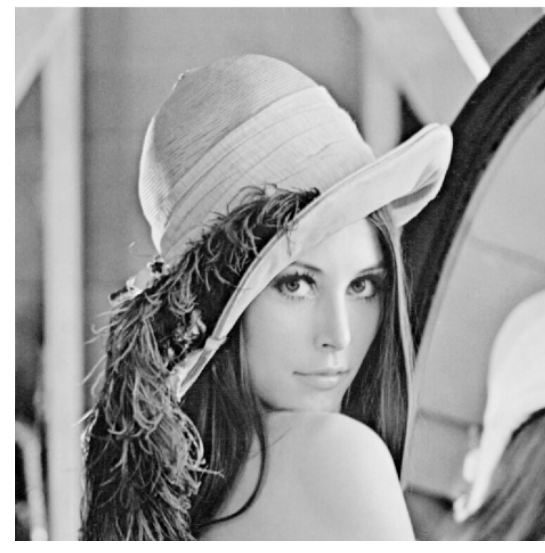

(a)

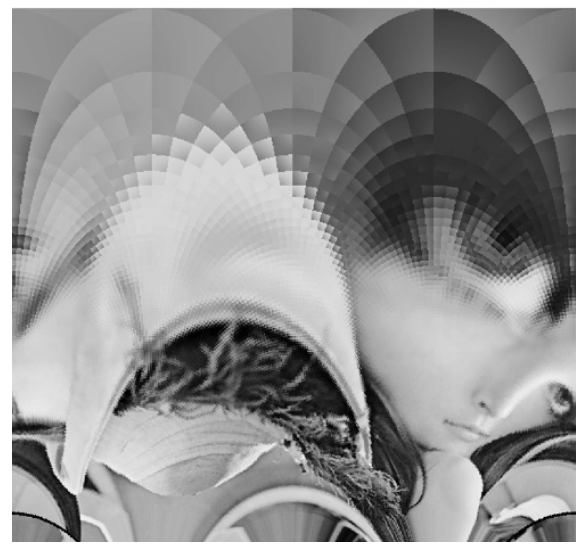

(b)

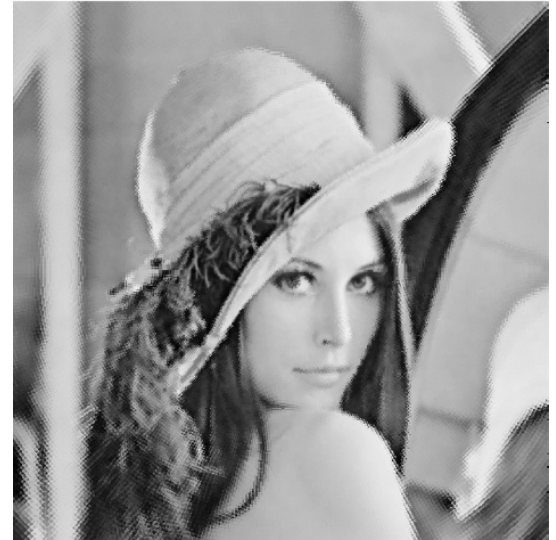

(c)

FIGURE 1: Distortions introduced by the log-polar map. (a) Original image Lena, (b) log-polar map, and (c) recovered image from the log-polar map.

for decoding is lost. To deal with these attacks, a spatial watermark is more appropriate as it targets specific locations in the image. Watermarking in the spatial domain is less resilient to common image processing operations, since the watermark becomes undetectable when the intensity information is modified. However, spatial techniques allow relevant information relating to the spatial distribution of pixels to be extracted. With this information, following geometric transformations such as rotation, the image can be resynchronized. The most common strategy for detecting a watermark after geometric distortion is to try to identify what the distortions are and then to invert them before applying the detector, for example, by introducing a template $[7,8]$. This requires the insertion and the detection of two watermarks: one of which does not carry information but helps to detect geometric transformations and a second one in which the hidden information is represented. This approach has two drawbacks: it further affects image fidelity and it increases the probability of false negatives. Additionally, in general this technique requires exhaustive searches thus resulting in a significant increase of workload. Since the watermarking strategy should be public, it is rather easy to destroy the synchronization template.

A more elegant approach to achieve robustness against loss of synchronization is to use transformations that map the signal information into an invariant domain. The most commonly used transformations depend on the properties of the Fourier transform. They use well-established techniques in pattern recognition and the invariance properties of the Fourier-Mellin transform [9]. Combining the Fourier transform with a log-polar map result on invertible rotation, translation, and scale invariant representation. Nevertheless, as noted by, Ruanaidh and Pun [10] using this to achieve watermarking leads to difficulties. The main problem is that the exponential nature of the inverse log-polar mapping causes a loss of image information in the discrete space.

To illustrate this problem, in Figure 1 the original image Lena along with the log-polar representation and the recon- structed image using the inverse log-polar mapping is shown. Although in these images a high resolution is used, the loss of image quality in the reconstructed image is clearly visible. Another problem is that achieving high resolution in the log-polar space requires interpolation. This causes further numerical instabilities since interpolation behaves badly if the interpolation nodes are not of the same scale [10]. Recently, Lin et al. [11] proposed another approach based on the Fourier-Mellin transform. The main idea in this technique is to use a noninvertible extraction function in order to overcome the instabilities arising when strong invariant representations are used. Although this approach tackle some implementation problems arising from the nature of the Fourier-Mellin transform, other aspects of robustness remain unsolved. For instance, strong resilience against image processing operations like JPEG compression is severely affected using this technique.

The main goal of this research is to overcome the robustness limitations of reported watermarking methods. We propose to combine frequency and spatial domain watermarking strategies into a new model in which the different robustness properties of both schemes are consistently exploited. The frequency domain is used to hide the watermark in the host image. DCT coefficients from the middle frequency bands are selected and a sequence of pseudo-random real numbers are inserted within these coefficients. Basically, this is a well-established approach proven to be extremely robust against additive noise, digital-analog-digital conversion and compression. These are the required properties of any watermarking scheme that we want to keep. To maximize robustness and minimize the introduced distortions, the pseudorandom watermark signal is adaptive spreading in the frequency domain. In this process the distortion amplitude is defined adaptively according to the shape of a just noticeable distortion mask. The just noticeable distortion (JND) is estimated from the characteristics of the image content. Finally, invariant primitives extracted from the pixel domain are used to achieve synchronization in case of geometric 


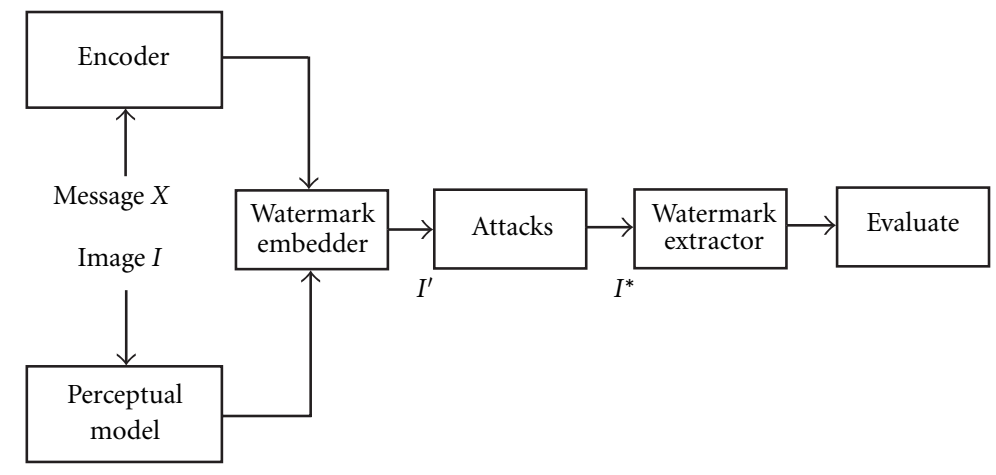

FIgure 2: System overview.

attacks. The proposed technique consists of three basic steps: (1) content based estimation of JND in the frequency domain; (2) adaptive spreading of a pseudo-random watermark signal in the frequency domain; and (3) extraction of relevant information related to the spatial distribution of pixels in the original image in order to resynchronize a geometric distorted image. The useful combination of a frequency domain watermarking approach with spatial information leads to a very robust system. The proposed system benefits from the advantages of both spread spectrum and image primitive invariance with respect to affine image transformations. In Figure 2, an overview of the implemented system is given.

In the first step, the image is analyzed in both the frequency and the spatial domain in order to detect the distortion sensitivity of the image according to its content. Local information derived from texture, edge, and luminance information is used to define a measure of JND. The JND is used adaptively according to the image content to maximize the amount of information (signal) that will be embedded as the watermark. To insert the watermark signal the middle DCT-frequency band of a block-wise transformed image is used. To maximize the capacity a pseudo-random signal with amplitude just below the image distortion, sensitivity is created according to the JND mask. Thus, the watermark signal is spread over the whole host by keeping its amplitude below the noise sensitivity of each pixel.

By transforming the original image into a suitable frequency domain information, redundancy of the spatial domain can be highly decorrelated and high-energy compaction can be achieved. The popular DCT transform for coding and compression consists of dividing the image into smaller blocks of the same size, and then transforming each block to obtain equal-sized blocks of transform coefficients. These coefficients are then thresholded and quantized in order to remove subjective redundancies. Since one of the most common and useful image processing methods is compression, it seems reasonable to exploit the knowledge about the processing methodology to generate a watermark scheme resilient to this image modification. Basically, we use the middle DCT-frequency band of a block-wise transformed image to insert the watermark signal. To maximize capacity, a pseudo-random signal with amplitude just below the image distortion sensitivity is created according to the JND mask.
Thus, the watermark signal is spread over the whole host by keeping its amplitude below the noise sensitivity of each pixel.

The spatial domain is used to resynchronize the image. This is implemented by applying primitive matching using point characterization according to Hilbert differential invariants [12]. These invariants have been defined in the literature for gray-level images, where good results can be obtained using differentials up to order three. For colour images this characterization can be improved if the three colour planes are used. In this case, only first-order invariants are necessary. Using the colour information a more efficient characterization can be achieved, as only first-order derivatives are necessary. In this work a generalization of the Harris corner detector [13] is used to detect salient image points. Furthermore first-order Hilbert invariants [12] are selected to characterize the salient image primitives. Finally, to detect the affine transformations undergone by the attacked image, a matching technique is used in which the extracted image primitives are compared.

The paper is organized as follows: in Section 2, the method for extraction of the JND mask is described. Section 3 deals with the watermark insertion and extraction in the DCT domain. Correction of geometric distortions using Hilbert invariants is described in Section 4. To assess the performance of the proposed watermarking method, several experiments have been conducted. The obtained results show that, this approach is resilient to most common distortions due to image processing transformations including high compression, geometrical distortions as well as some malicious attacks. Selected results of computer simulations are reported in Section 5. The paper is closed with conclusions in Section 6.

\section{JND-MASK GENERATION}

The process of embedding a watermark in any image can be regarded in the same way as adding noise to the image. This process leads to an alteration of the host image. Obviously, altering a large number of pixel values arbitrarily will result in noticeable image distortions. These distortions are proportional to the amplitude of the embedded signal. Consequently, an image can be distorted only to a certain limit 


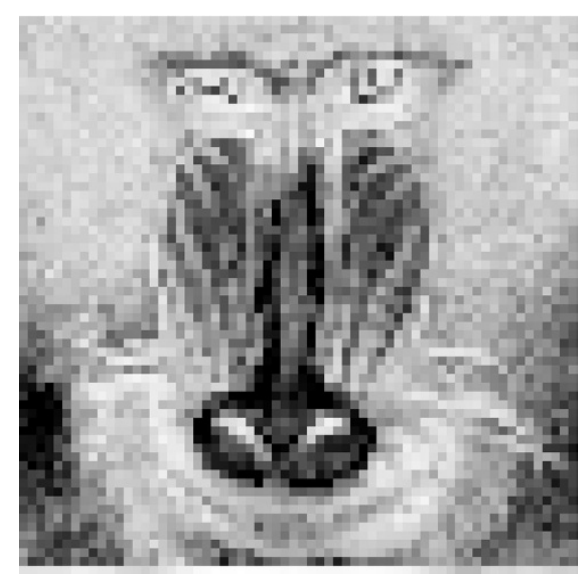

(a)

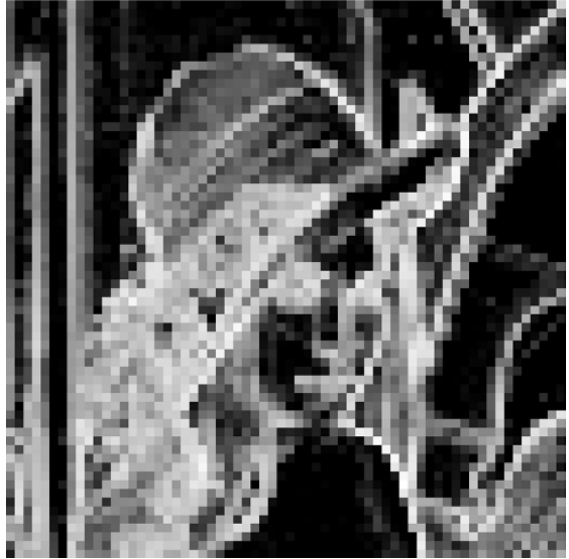

(b)

Figure 3: $D_{T}$ vales for Baboon (a) and Lena (b).

without making the difference between the original and the altered one perceptible. This limit varies according to the image content and is called just noticeable distortion (JND). Several methods can be found in the literature to estimate JND masks in the context of compression and image quality assessment. Several perceptually-based algorithms have been proposed in the literature [14]. An overview of visual models for signal compression is presented in [15]. Most of these models use both frequency sensitivity, and spatial masking based on image edges. The spatial masking is a modified version of the spatial masking model presented in [16]. The main principle of the spatial masking is that, edges in an image are able to mask signals of much greater amplitude than regions of near-constant intensity. For a given image, a tolerable-error level value may be formed for each pixel.

To estimate the JND mask, three image characteristics are considered: texture, edgeness, and smoothness. According to several studies about the human vision system, it is well known that the distortion visibility in highly textured areas is very low. This means that these areas are the most suitable ones to hide the watermark signal. In fact textured regions present a very high noise-sensitivity level. In contrast to that, edge information of an image is the most important factor for the human image perception. Consequently, edges have the least noise sensitivity and it is essential to keep edge integrity in order to preserve the image quality. Edges have the lowest JND values. Similarly, smooth image areas have a general bandpass characteristic. They influence the human perception and consequently their JND values are also relatively low. The definition of a suitable JND mask depends essentially on the accurate extraction of image texture, edges, and smooth areas.

Texture information can be retrieved directly from the transformed domain by analyzing the DCT coefficients. Following the JPEG and MPEG2 encoding strategies, the image is first split into $8 \times 8$ blocks. Each block is transformed in the DCT domain and the resulting 64 coefficients analyzed. It is well known that in high textured regions or along edges, the signal energy concentrates in the high frequency coefficients while in uniform image areas the signal energy is concentrated in the low frequency components. Thus, the energy of the AC coefficients can be used as a measure for texture within each block. Using the following formula for the energy in the AC coefficients a measure for texture $D_{T}$ within each block is derived:

$$
D_{T}=\log \left(\sum_{i=1}^{63} v_{i}^{2}-v_{0}^{2}\right),
$$

where $v_{i}, i=0, \ldots, 63$, are the 64 DCT coefficients of the considered block. The obtained $D_{T}$ values are scaled to the range $[0,64]$ and the resulting normalized values assigned to the corresponding blocks. The images in Figure 3 show the obtained $D_{T}$ values for the two test images Lena and Baboon. In this representation the $D_{T}$ values have been scaled to the range $[0,255]$ and rounded to the nearest integer.

Edges and smooth areas are extracted from the pixel domain. The main difficulty here consists of discriminating between relevant image edges and spurious edges due to noise and texture. Relevant edges are detected using either the Canny operator. The length of each single edge is calculated by traversing it. Edges whose length does not exceed a threshold are considered texture edges and are removed. Using the binary image containing the final edge information, edgeness is calculated block-wise according to the formula

$$
D_{E}=\frac{64 \cdot P_{E}}{\max \left(P_{E}\right)}
$$

where $P_{E}$ is the cardinality of the set of pixels within the block and at edge locations and $\max \left(P_{E}\right)$ is the maximum value of $P_{E}$ over all the blocks in the image.

Finally, the Moravec operator is used to extract uniform image regions. The same strategy introduced in [17] for the recognition of uniform regions is used. To avoid the effects of noise, the Moravec operator is applied on the Gaussian 


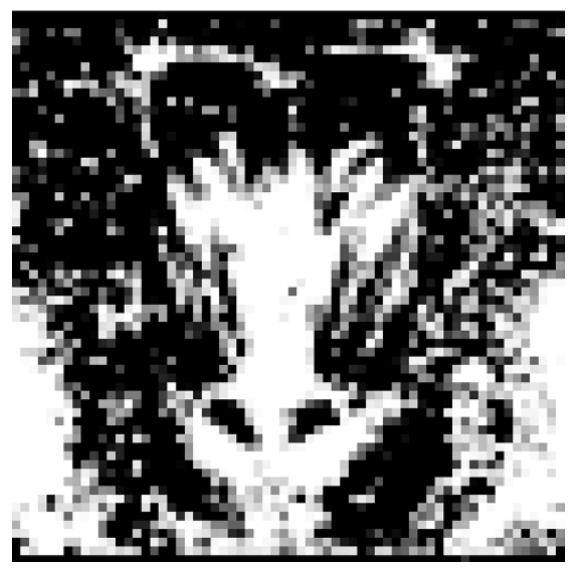

(a)

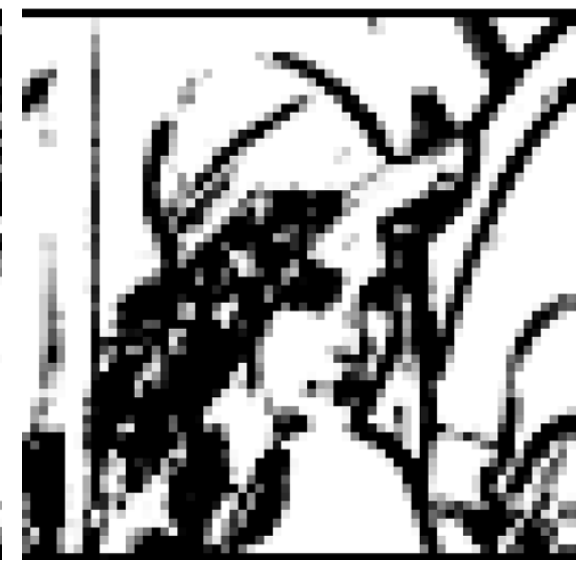

(b)

Figure 4: Extracted $D_{U}$ vales for Baboon (a) and Lena (b).

smoothed image. Additionally, small picks are removed using a median filter over the binary image obtained after thresholding. The uniformity $D_{U}$ in a block is defined as the number of pixels belonging to a uniform area within the block. The images in Figure 4 show the obtained $D_{U}$ values for the two test images Lena and Baboon. In this representation the $D_{U}$ values have been scaled to the range $[0,255]$ and rounded to the nearest integer.

Using the three values $D_{T}, D_{E}$, and $D_{U}$ an initial estimate for the JND value over each block is calculated as

$$
\tilde{J}=D_{T}-\frac{1}{2}\left(D_{E}+D_{U}\right) .
$$

Since the human vision system is more sensitive to intensity changes in the mid-gray region and its sensitivity fail parabolically at both ends of the gray scale, a correction to $\tilde{J}$ is used. The final JND values are obtained as $J=\tilde{J}+(128-\tilde{I})^{2}$, where $\tilde{I}$ is the average of the luminance values within the considered block.

\section{WATERMARK EMBEDDING IN THE DCT DOMAIN}

The method of watermark insertion is to modify selected DCT coefficients by embedding a sequence of pseudorandom numbers within them. This technique does not target individual pixels but rather, upon inverse transformation, the watermark will be dispersed over the entire image. The watermark itself consists of a sequence of real pseudorandom numbers $X=x_{1}, \ldots, x_{m}$, with $m \leq 64$, satisfying the normal distribution $\eta(0,1)$. The array of DCT coefficients obtained from the transformation consists of a sequence of values $V=v_{1}, \ldots, v_{64}$.

The watermark $X=x_{1}, \ldots, x_{m}$ is inserted into $m$ selected coefficients from the middle frequency bands. This yields an adjusted set of values $V^{\prime}=v_{1}^{\prime}, \ldots, v_{64}^{\prime}$. The inverse of the DCT is then performed to obtain the watermarked image $I^{\prime}$. To insert the watermark we use the formula

$$
v_{i}^{\prime}=v_{i}+\alpha \cdot J \cdot\left|v_{i}\right| x_{i}
$$

where $\alpha$ is a scaling parameter and $J$ is the distortion parameter previously defined. If the watermarked image $I^{\prime}$ is transformed, by processing or attacking, a new image $I^{*}$ is generated.

The presence of the watermark can be detected in the transformed image $I^{*}$ by performing a correlation test

$$
R=\frac{1}{N}\left(V^{*} \cdot X\right),
$$

where $V^{*}$ is the vector containing those extracted DCT coefficients which have been modified and $X$ is the original watermark. Since this is a statistical test we must be aware of the possibility of obtaining detection errors. To decide whether the watermark is authentic, we must determine some threshold $T$ and test whether the obtained correlation coefficient is greater than $T$. Setting the detection threshold is a decision based on the desire to minimize both false positives and false rejections.

Figure 5a shows the watermarked image using this spread spectrum technique. Although high distortions have been introduced to some DCT values according to the JND mask the image quality has not been compromised. In Figure 5b the detector response is shown for the same image after JPEG compression using 50\% quality factor. In Figure 6, similar results are shown after digital-analog-digital conversion.

\section{GEOMETRIC ATTACKS AND RESYNCHRONIZATION}

Since most digital images are coloured rather than gray-level, the entire colour information can be exploited using wellknown gray-level image attributes independently for each colour plane. We are interested in attributes that are invariant with respect to as large a group of geometric transformations as possible, but specifically in orthogonal and affine transformations. The study of these invariants can be traced back to the 18th century in the work undertaken by the renowned 


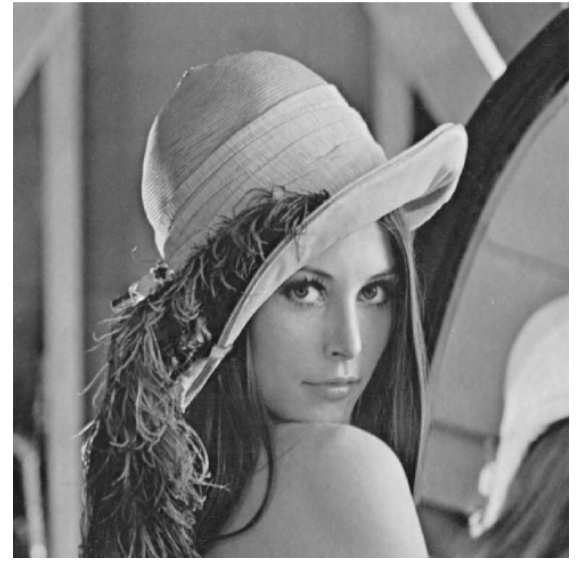

(a)

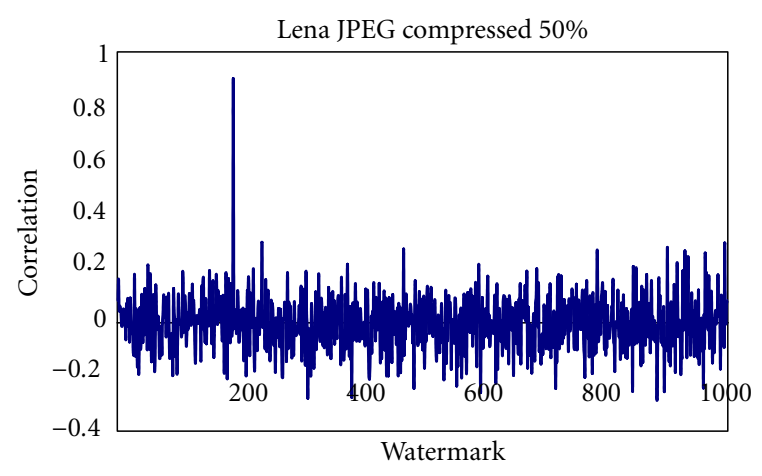

(b)

FIgURE 5: Watermarked image and response of the watermark detector after JPEG-compression using 50\% quality factor to 1000 randomly generated watermarks including the genuine watermark at position 188 .

mathematician David Hilbert [12]. He showed that any invariant of finite order can be expressed as a polynomial function of a set of irreducible invariants. Although the invariant theory has been widely applied in pattern recognition and computer vision [18], it has not been used to recover transformation parameter and resynchronize signal in watermarking applications. In a gray-level image the fundamental set of irreducible invariants can be defined as $I, I_{\eta}, I_{\eta \eta}, I_{\varsigma \eta}$, $I_{\varsigma \varsigma}$, with the unit vector $\eta$ given by $\eta=\nabla I /|\nabla I|$ and $\varsigma \perp \eta$.

Given an original image $I$, the first step in generating a feature space with invariant attributes is to detect some key points that are robust to common image processing and geometrical transformations. We have chosen the Harris detector which uses only first-order derivatives and is well known as one of the most stable and robust corner detectors in image processing. The Harris detector is defined as the positive local extreme of the following operator:

$$
\operatorname{Det}(M)-k \operatorname{Trace}^{2}(M)
$$

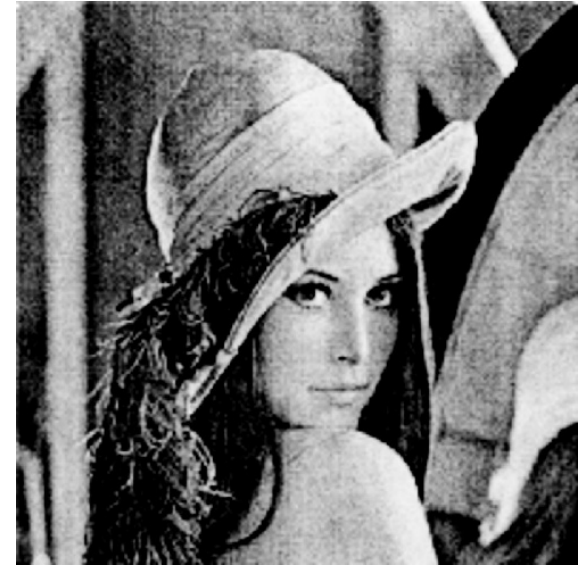

(a)

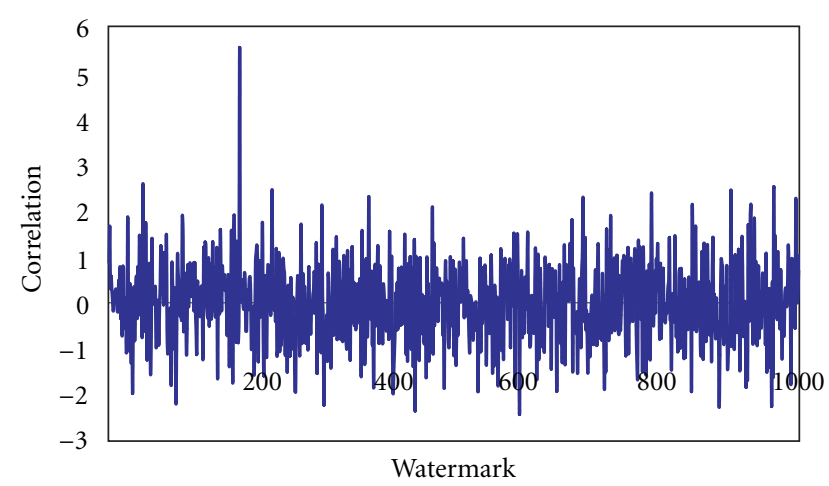

(b)

FIGURE 6: (a) Image obtained after printing, photocopying, and scanning the watermarked Lena and (b) response of the watermark detector to 1000 randomly generated watermarks including the genuine watermark at position 188 .

where

$$
M=\left[\begin{array}{ll}
C_{11} & C_{12} \\
C_{21} & C_{22}
\end{array}\right],
$$

with $C_{11}=\Gamma \sigma\left(R_{x}^{2}+G_{x}^{2}+B_{x}^{2}\right), C_{12}=\Gamma \sigma\left(R_{x} \cdot R_{y}+G_{x} \cdot G_{y}+B_{x} \cdot B_{y}\right)$, $C_{21}=\Gamma \sigma\left(R_{x} \cdot R_{y}+G_{x} \cdot G_{y}+B_{x} \cdot B_{y}\right), C_{22}=\Gamma \sigma\left(R_{y}^{2}+G_{y}^{2}+B_{y}^{2}\right)$, $k=0.04$ is a scalar, and $\Gamma$ is the Gaussian convolution kernel of variance $\sigma$.

As mentioned before the basic idea behind the strategy for defining an invariant feature space is to use first-order Hilbert invariants. For each corner point, the vector comprising the following eight differential primitives is calculated:

$$
F=\left(R,|\nabla R|^{2}, G,|\nabla G|^{2}, B,|\nabla B|^{2}, \nabla R \cdot \nabla G, \nabla R \cdot \nabla B\right)^{T} .
$$

The vector (8) forms the space of feature invariants in the considered colour image. It is invariant with respect to rotation, translation, and scaling which are the most common 


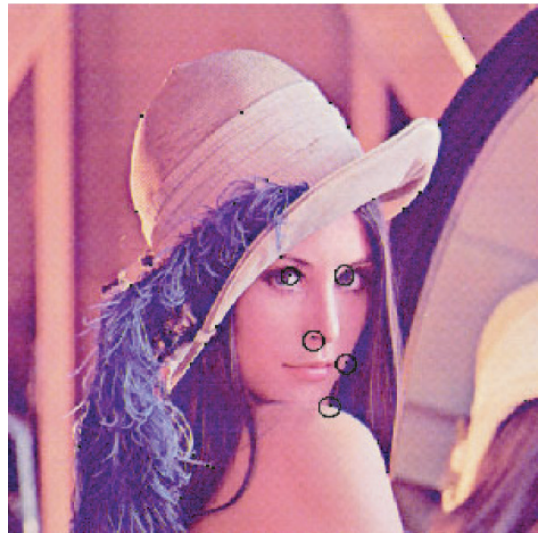

(a)

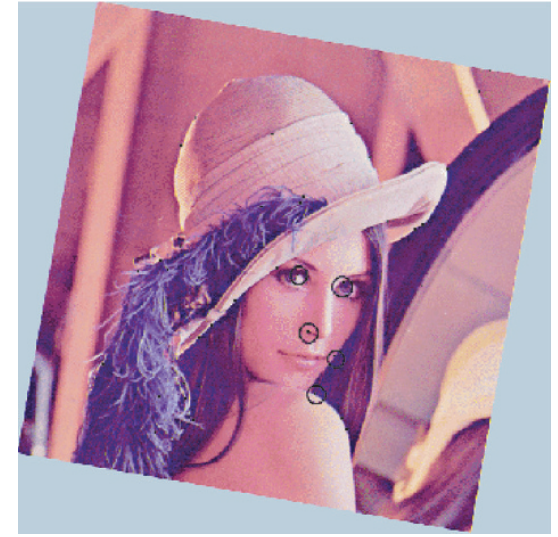

(b)

Figure 7: (a) Selected relevant corners using the Harris detector and (b) matched corners using first order Hilbert invariants.

geometric transformations. It allows very robust characterization with regard to noise, since only first-order derivatives are involved. Additionally, the complexity of the method remains very low since only simple pixel differences should be calculated. The use of higher-order derivatives involves heavier workload and would cause severe instabilities. Finally, the transformation parameters are detected by matching the corners in the primitive space defined by (8). In the matching procedure the conventional Euclidean distance between the primitives is used as similarity measure.

\section{EXPERIMENTAL RESULTS}

Several experiments have been conducted to test the performance of the proposed system. In this section some selected results are reported. The images in Figures 5 and 6 show the watermarked image Lena after compression and format conversion as well as the detector response obtained using the technique described in Section 3.

Tests against geometric attacks such as cropping, rotations, scaling, and so forth, have been carried out. In most cases, good synchronization has been achieved. For cropping attacks the image must be padded to the original size before decoding. Thus frequency sampling is performed with the same step both by the encoder and the decoder. In this case, cropping robustness is obtained from the invariance of the magnitude spectrum to spatial shifting. In all cases, the parameters describing the performed geometrical attack have been detected using the technique described in Section 4 . Figure 7 shows the detected corners in the watermarked image. In this representation corners are highlighted as small black spots. For the sake of visibility in this representation, five corner points appear surrounded by circles. The image in Figure $7 \mathrm{~b}$ has been rotated clockwise by 15 degrees. The corresponding corners are also represented by small black spots. Matching between image primitives using the Hilbert invariant features, described in Section 4, reveal the parameters encoding the geometric transformations undergone by

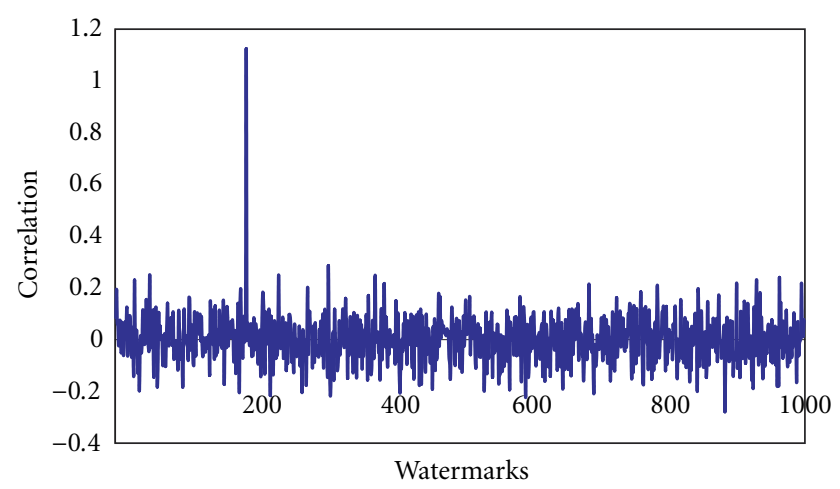

Figure 8: Response of the watermark detector after re-synchronization.

the attacked image. In this case then rotation angle has been recovered accurately.

The recovered parameters have been used to re-synchronize the transformed image and to detect the watermark in the DCT domain. Figure 8 shows the response of the watermark detector for the rotated image in Figure 7b. Using the technique described in Section 4, the rotation angle has been estimated and the attacked image rotated back. The watermark detector has been applied to the resynchronized image.

\section{CONCLUSIONS}

First-order invariant image primitives have been combined with a spread spectrum approach to produce a novel watermarking scheme. In the introduced technique robustness against usual image processing and distortions is achieved by combining a well-established spread spectrum approach with an adaptive masking of the JND. The JND is extracted from the image content itself. Unlike most common strategies that use a reference template to detect geometrical distortions, the schema presented in this paper exploit relevant 
image features to perform resynchronization. The spatial domain is used to extract relevant information related to the pixels distribution in the original image. The underlying strategy consists of characterizing salient image points using first-order differential invariants. These primitives are invariant to orthogonal image transformations. To detect geometrical attacks in a frequency-domain watermarked image a robust matching technique is applied. Features extracted from the original image are matched with those extracted from the attacked image. Their disparities reveal the transformation parameters and are used to re-synchronize the attacked image. Experimental results confirm the robustness of the proposed schema.

\section{REFERENCES}

[1] Signal Processing, "Special Issue on Watermarking," vol. 66, no. 3, 1998.

[2] Proceedings of the IEEE, "Special Issue on Identification and Protection of Multimedia Information," vol. 87, no. 7, 1999.

[3] C.-T. Hsu and J.-L. Wu, "Hidden digital watermarks in images," IEEE Trans. Image Processing, vol. 8, no. 1, pp. 58-68, 1999.

[4] R. B. Wolfgang, C. I. Podilchuk, and E. J. Delp, "Perceptual watermarks for digital images and video," Proceedings of the IEEE, vol. 87, no. 7, pp. 1108-1126, 1999.

[5] M. Barni, F. Bartolini, V. Cappellini, A. Lippi, and A. Piva, "A DWT-based technique for spatio-frequency masking of digital signatures," in Security and Watermarking of Multimedia Contents, Electronic Imaging, vol. 3657 of Proceedings of SPIE, pp. 31-39, San Jose, Calif, USA, January 1999.

[6] A. Piva, M. Barni, F. Bartolini, and V. Cappellini, "DCT-based watermark recovering without resorting to the uncorrupted original image," in Proc. 4th IEEE International Conference on Image Processing (ICIP '97), vol. 1, pp. 520-523, Santa Barbara, Calif, USA, October 1997.

[7] S. Pereira and T. Pun, "Fast robust template matching for affine resistant image watermarking," in International Workshop on Information Hiding, vol. 1768 of Lecture Notes in Computer Science, pp. 200-210, Springer-Verlag, Dresden, Germany, 29 September-1 October 1999.

[8] M. Kutter, "Watermarking resistance to translation, rotation, and scaling," in SPIE Conf. on Multimedia Systems and Applications, vol. 3528, pp. 423-431, 1998.

[9] R. D. Brandt and F. Lin, "Representations that uniquely characterize images modulo translation, rotation, and scaling," Pattern Recognition Lett., vol. 17, no. 9, pp. 1001-1015, 1996.

[10] J. J. K. O'Ruanaidh and T. Pun, "Rotation, scale and translation invariant spread spectrum digital image watermarking," Signal Processing, vol. 66, no. 3, pp. 303-318, 1998.

[11] C.-Y. Lin, M. Wu, J. A. Bloom, M. L. Miller, I. J. Cox, and Y. M. Lui, "Rotation, scale, and translation resilient watermarking for images," IEEE Trans. Image Processing, vol. 10, no. 5, pp. 767-782, 2001.

[12] D. Hilbert, Theory of Algebraic Invariants, Cambridge Mathematical Library, Cambridge University Press, Cambridge, 1890.

[13] C. Harris and M. Stephens, "A combined corner and edge detector," in Proc. 4th Alvey Vision Conference, pp. 147-151, Manchester, 1988.

[14] I. S. Hontsch and L. J. Karam, "APIC: adaptive perceptual image coding based on sub-band decomposition with locally adaptive perceptual weighting," in Proc. 1997 IEEE International Conference on Image Processing (ICIP '97), vol. 1, pp. 37-40, Santa Barbara, Calif, USA, October 1997.
[15] N. S. Jayant, J. D. Johnston, and R. Safranek, "Signal compression based on models of human perception," Proceedings of the IEEE, vol. 81, no. 10, pp. 1385-1422, 1993.

[16] B. Girod, "The information theoretical significance of spatial and temporal masking in video signals," in Proc. SPIE Human Vision, Visual Processing, and Digital Display, vol. 1077, pp. 178-187, Los Angeles, Calif, USA, 1989.

[17] E. Izquierdo, "Stereo image analysis for multi-viewpoint telepresence applications," Signal Processing: Image Communication, vol. 11, no. 3, pp. 231-254, 1998.

[18] P. Montesinos, V. Gouet, R. Deriche, and D. Pelé, "Matching color uncalibrated images using differential invariants," Image and Vision Computing, vol. 18, no. 9, pp. 659-671, 2000.

Ebroul Izquierdo is a Lecturer in the Department of Electronic Engineering, Queen Mary University of London. He received the Dr. Rer. Nat. from the Humboldt University, Berlin, Germany, in 1993. From 1990 to 1992 he was a Teaching Assistant at the Department of Applied Mathematics, Technical University, Berlin. In 1993, Dr. Izquierdo joined the Heinrich-Hertz Institute for Communication Technology

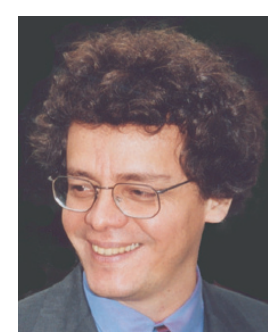
(HHI), Berlin, Germany, where he worked from 1993 to 1997. During this period he developed and implemented techniques for stereo vision, disparity and motion estimation, video compression, 3D modelling, and immersive telepresence. From 1998 to 1999, Dr. Izquierdo was with the Department of Electronic Systems Engineering of the University of Essex, UK as a Senior Research Officer. He worked on research dealing with content-based coding, efficient indexing, and retrieval of video and nonlinear diffusion models for image processing. Dr. Izquierdo has been involved in research and management of projects in Germany, the UK, and two European projects in the multimedia field. In 1999, Dr. Izquierdo was awarded a short term British Telecom fellowship. Currently, he is the coordinator of the European IST project BUSMAN and represents QMUL in the European IST Network of Excellence SCHEMA. Dr. Izquierdo is an IEE Chartered Engineer, a member of the IEEE, the IEE, and the British Machine Vision Association. He represents the UK in the European COST211 forum and is member of the management committee of the Information Visualization Society. He is an active member of the Programme Committee of the IEEE Information Visualization, the EURASIP \& IEEE International Conference on Video Processing and Multimedia Communication and the COST211 sponsored European Workshop on Image Analysis for Multimedia Interactive Services. He has published over 100 papers including chapters in books. 